\title{
Is the Poverty Gap a Barrier to Early Initiation of Breastfeeding in Rural Niger?
}

\section{Naoko Horii*}

Department of Behavior Change Communication, Maternal Child Health and Nutrition, France

*Corresponding author: Naoko Horii, Independent Consultant of Behavior Change Communication, Maternal Child Health and Nutrition, France, Tel: +33-695134523; E-mail: Naoko.Horii@gmail.com

Received date: July 20, 2016; Accepted date: August 04, 2016; Published date: August 09, 2016

Copyright: (c) 2016 Horii N. This is an open-access article distributed under the terms of the Creative Commons Attribution License, which permits unrestricted use, distribution, and reproduction in any medium, provided the original author and source are credited.

\section{Short Communication}

There is strong evidence about the benefits of early, exclusive and continued breastfeeding to mitigate maternal and child mortality risk and illnesses [1-4]. Breastfeeding has been globally recognized as a measurement indicator for the health outcome of populations [5] However, a secondary analysis of the Niger 2012 demographic health survey showed that exclusivity of postpartum breastfeeding was compromised too early by prelacteal feeding: half of the interviewed women $(50.3 \%)$ reported having given their child other liquids than breast milk in the first 3 days of birth [6]. The $67^{\text {th }}$ World Health Assembly adopted a resolution on sustainable actions across sectors to improve health equity [7]. Community based interventions led by a non-health sector were recognized to improve neonatal health of the poorest [8]. Studies showed that early initiation of breastfeeding, a critical protective factor for neonatal survival, is impaired by socioeconomic vulnerability of mothers [9-11]. A recently published article by the author clearly revealed the importance of identifying risk factors hindering early initiation of breastfeeding for which the scientific evidence remains scarce in Sub-Saharan Africa [12].

A cross-sectional quantitative study was conducted to evaluate a UNICEF led behavior change communication (BCC) program promoting community based child health care in rural Niger. The uniqueness of this study underpinned intra-family interactions, community involvement of the interviewed women and their families in neonatal and child health care promotion. This post-intervention survey included behavioral indicators known to determine the timing of initiating breastfeeding after birth at family and community levels. It analyzed BCC program strategies led by various actors selected as communication channels to interact directly or indirectly with the interviewed mothers to promote family do-able child health care outside the health system.

Effects of breastfeeding promotion are difficult to measure and to sustain as a short and long term preventive measurement of neonatal and infant death and illnesses. Causal relations between early and exclusive breastfeeding after birth and neonatal survival and health are not always clear to mothers, traditional birth attendants and even to trained health workers. Transfer of knowledge into practice is possible if mothers make their own decision to change behavior and if there is a sustainable supportive environment within their family and community encouraging mothers to adopt early breastfeeding after birth $[13,14]$. The above study provided a few clues to understand what community based BCC strategy and action worked the best to promote early initiation of breastfeeding through statistical analysis of the postintervention survey datasets. It concluded that peer breastfeeding support to other women suggested a behavior change model as a response to socio-economic disparity.
In Sarkinyama village of Maradi region, one of the pilot areas covered by the BCC program promoting child health care, we held a participatory workshop with a group of mothers to share the findings of health behavioral outcomes that have been collected through a program based participatory monitoring led by trained community volunteers. Although the findings came from scientifically unreliable sources without rigorous study design and data collection methods, the processes of collecting information and involving mothers in discussing the findings contributed to enhancing the confidence in optimal breastfeeding practices as beneficial acts to their child health. After sharing visual images in form of histograms representing numbers of mothers practicing and not practicing each type of child health care, a few mothers started spontaneously sharing their experience about breastfeeding: one declared that the first child who was not breastfed often had diarrhea and was ill and that the second child put to the breast immediately after birth and exclusively breastfed was healthy, gaining more weight and growing faster than the first child. Another mother confessed she was not confident and reluctant about breastfeeding but after listening to this testimony, she felt like doing the same to follow the previous mothers' experience. This type of participatory community led social change approach illustrates an example of sustainable behavior change in early breastfeeding promotion regardless of socio-economic status of mothers.

Whereas individual counseling at antenatal care provided by health workers and home visits by community volunteers showed limited impact on promoting early initiation of breastfeeding among mothers deprived from accessing basic health care and hygiene facilities, peer breastfeeding support through active involvement of mothers reached socio-economically vulnerable populations [12]. This is a key to overcome discrepancy between the better-off and poor who are inclined to be excluded from accessing BCC program support $[15,16]$.

\section{Acknowledgement}

We would like to thank the UNICEF Niger country office and the UNICEF Western and Central Africa regional office for their technical, administrative and financial support for the community based action research on child health care promotion undertaken in rural Niger. Our special thanks go to the National Institute of Statistics in Niger for leading field based data collection of the post-intervention survey to evaluate the child health care interventions in collaboration with community based organizations, local authority members, community volunteers and many others who made a considerable contribution to the program.

\section{References}

1. Black RE, Victora CG, Walker SP, Bhutta ZA, Christian P, et al. (2013) Maternal and child under nutrition and overweight in low-income and middle-income countries. The Lancet 382: 427-451. 
Citation: Horii N (2016) Is the Poverty Gap a Barrier to Early Initiation of Breastfeeding in Rural Niger? J Neonatal Biol 5: 227. doi:

2. Bhutta ZA, Das JK, Rizvi A, Gaffey MF, Walker N, et al. (2013) Evidencebased interventions for improvement of maternal and child nutrition: What can be done and at what cost? Lancet 382: 452-477.

3. Debes AK, Kohli A, Walker N, Edmond K, Mullany LC (2013) Time to initiation of breastfeeding and neonatal mortality and morbidity: A systematic review. BMC Public Health 13: S19-S19.

4. Edmond KM, Zandoh C, Quigley MA, Amenga-Etego S, Owusu-Agyei S et al. (2006) Delayed breastfeeding initiation increases risk of neonatal mortality. Pediatrics 117: e380-386.

5. Jones CP, Jones CY, Perry GS, Barclay G, Jones CA (2009) Addressing the social determinants of children's health: A cliff analogy. J Health Care Poor Underserved 20: 1-12.

6. Horii N (2015) Postpartum breastfeeding in rural Niger: Demographic analysis of a communication program for child health care. CEPED, Paris Descartes.

7. World Health Organization (2014) Sixty-seventh World Health Assembly Agenda item 14.6 Contributing to social and economic development: Sustainable action across sectors to improve health and health equity. Geneva.

8. Lassi ZS, Bhutta ZA (2015) Community-based intervention packages for reducing maternal and neonatal morbidity and mortality and improving neonatal outcomes. Cochrane Database Syst Rev 3: CD007754.

9. Hailemariam TW, Adeba E, Sufa A (2015) Predictors of early breastfeeding initiation among mothers of children under 24 months of age in rural part of West Ethiopia. BMC Public Health 15: 1076
10. Lawoyin TO, Olawuyi JF, Onadeko MO (2001) Factors associated with exclusive breastfeeding in Ibadan, Nigeria. J Hum Lact 17: 321-325.

11. Waiswa P, Peterson S, Tomson G, Pariyo GW (2010) Poor new-born care practices - a population based survey in eastern Uganda. BMC Pregnancy Childbirth 10: 9 .

12. Horii N, Habi O, Dangana A, Maina A, Alzouma S, et al. (2016) Community-based behavior change promoting child health care: A response to socio-economic disparity. Journal of Health, Population and Nutrition 35: 1-10.

13. United Nations Children's Fund UNICEF (2008) Communication for Development (C4D) Strategic Framework and Plan of Action 2008-2011.

14. Andrien M, Beghin I (1993) Nutrition et communication de leducation nutritionnelle conventionnelle a la communication sociale en nutrition. Harmattan, Paris.

15. Callaghan-Koru JA, Nonyane BAS, Guenther T, Sitrin D, Ligowe R, et al. (2013) Contribution of community-based new-born health promotion to reducing inequities in healthy new-born care practices and knowledge: Evidence of improvement from a three-district pilot program in Malawi. BMC Public Health 13: 1052.

16. Fenn B, Kirkwood BR, Popatia Z, Bradley DJ (2007) Inequities in neonatal survival interventions: Evidence from national surveys. Arch Dis Child Fetal Neonatal Ed 92: F361-366. 